\title{
AOTrauma-D-A-CH-Reisestipendium 2017
}

Markus Heinecke

\author{
Die 3 AOTrauma-Sektionen von Deutschland (D), Österreich (A) und der Schweiz \\ $(\mathrm{CH})$, abgekürzt D-A-CH, haben im Jahr 2017 zum 1. Mal jeweils pro Land ein Reise- \\ stipendium ausgeschrieben. Das Ziel ist, den Austausch von klinisch aktiven Ärzten \\ zwischen den 3 Ländern zu fördern. Dr. Markus Heinecke erhielt das Stipendium aus \\ Deutschland und ging zu Dr. Christoph Sommer in die Schweiz.
}

\section{Erfahrungsbericht von \\ Dr. Markus Heinecke, Halle}

Dank der großzügigen Unterstützung durch die AOTrauma Deutschland erhielt ich die Möglichkeit, als AO-Gastarzt im März 2018 im Kantonsspital Graubünden (KSGR) hospitieren zu dürfen. Für mich eine einmalige Chance, einem der führenden Unfallchirurgen auf den Gebieten der gelenknahen Osteosynthesen, vor allem der unteren Extremität und der minimalinvasiven Operationstechnik, Dr. med. Christoph Sommer, über die Schulter zu schauen. Und um eines vorweg zu nehmen: Meine Erwartungen wurden mehr als übertroffen. Dank perfekter Organisation meines gesamten Aufenthaltes in Chur durch den betriebswirtschaftlichen Mitarbeiter des KSGR, Marco Rettich, fühlte ich mich vom ersten Tag an herzlich willkommen und war so schnell in den Abläufen und vor allem im OP-Alltag integriert.

Das Kantonsspital Graubünden in Chur ist das Zentrumsspital in der Südostschweiz mit 3 nahegelegenen Standorten, wobei ich mich die meiste Zeit im Hauptstandort befand. Die Abteilung für Allgemein- und Unfallchirurgie wird durch Chefarzt Dr. Sommer geleitet und von einem überaus herzlichen Team von Kolleginnen und Kollegen vervollständigt, die seine AO-treuen Prinzipien ebenfalls verinnerlicht haben. Durch die gute Lage von Chur zu einigen großen Skigebieten handelt es sich bei den Verletzten in den Wintermonaten vielfach um verunfallte Skioder Snowboardfahrer in allen Altersklassen sowie Patienten mit Unfällen vor allem aus anderen Freizeitsportarten. So ist die unfallchirurgische Abteilung täglich mit schwerwiegenden Frakturen und auch Mehrfachverletzten konfrontiert.

Vor allem das dezidierte Auseinandersetzen mit jeglicher Fraktursituation unter Beachtung der Weichteile sowie das „Zeitnehmen“ für jeden einzelnen Patienten imponierte mir während meines gesamten Aufenthaltes sehr. Meine Zeit war durch das tägliche Visitieren, das Assistieren bei traumatologischen Eingriffen mit teils sehr komplexen Osteosynthesen und das Teilnehmen an der unfallchirurgischen Sprechstunde gekennzeichnet. Auch darüber hinaus nahm sich Dr. Sommer ausgiebig Zeit für mich, um spannende Fälle zu diskutieren und meine vielen, vielen Fragen zu beantworten. Außerdem erhielt ich

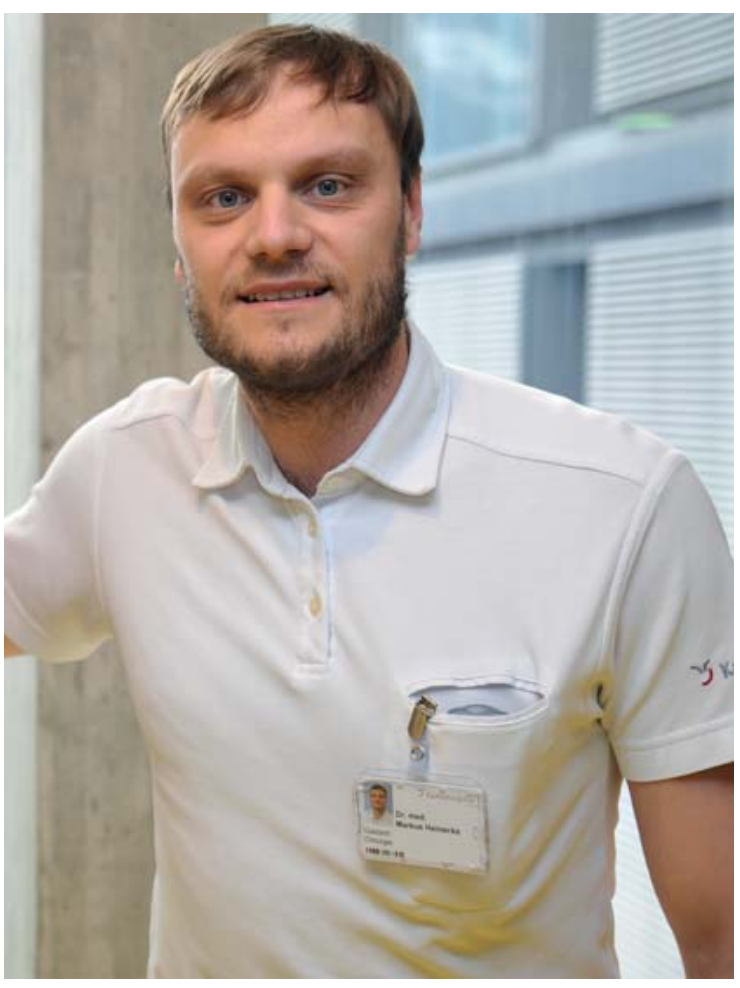

- Abb. 1 Markus Heinecke im Kantonsspital Graubünden. 


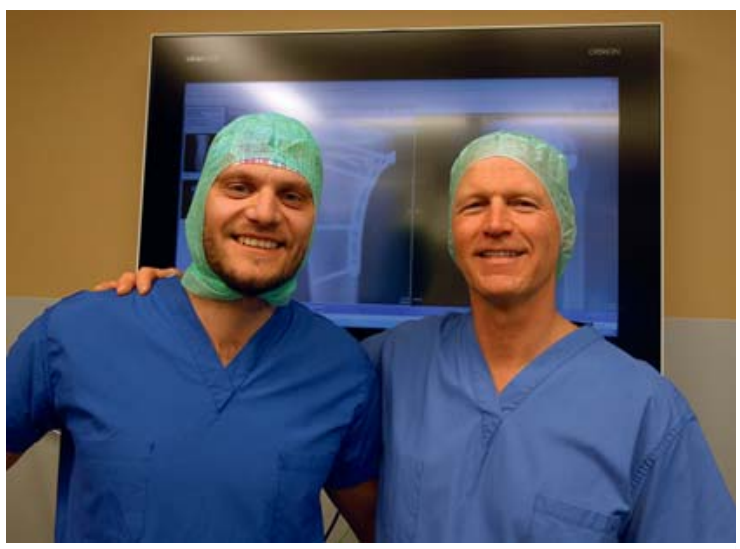

- Abb. 2 Markus Heinecke und Christoph Sommer.

Einblick in seine unglaublich umfangreiche Fallsammlung. Es ist wirklich beeindruckend, mit welcher Hingabe und Perfektion er seine Osteosynthesen plant und ausführt. Das Miterleben, wie er eine Operationsstrategie entwickelt und diese mit seinen Kolleginnen und Kollegen bespricht, ist einmalig und ein großer Zugewinn mit einem enormen Lerneffekt für mich gewesen.

Aber auch außerhalb des Klinikalltages wurde ich vom Team mit eingebunden. So gab es in meiner 2. Woche den jährlichen Skitag der chirurgischen Abteilung. Zu die- sem wurde ich herzlich eingeladen. Es gab sogar ein Skirennen mit professioneller Zeitmessung. Es wurde gefeiert, bis am späten Abend in der Dunkelheit mit Stirnlampen wieder ins Tal gefahren wurde. Ansonsten konnte ich dankenswerterweise durch den Leitenden Arzt der Unfallchirurgie, Dr. Thomas Müller, die Stadt Chur und seine Umgebung genauer kennenlernen und musste feststellen, dass es sehr lohnenswert erscheint, in dieser Region mit ihrer Vorzügen nicht nur zu arbeiten, sondern auch mit der Familie zu leben.

Insgesamt war die Zeit als AO-Gastarzt im KSGR für mich in der fachlichen und persönlichen Entwicklung mehr als gewinnbringend. Die gesammelten Erfahrungen werden mich zukünftig prägen und lassen mich immer wieder gerne an diese einmalige Zeit zurückdenken. Für die Gelegenheit der Hospitation im Kantonsspital Graubünden in Chur bei Chefarzt Dr. Sommer über das erhaltende Reisestipendium kann ich mich nur nachdrücklich bei der AOTrauma Deutschland bedanken.

Dr. Markus Heinecke, BG Klinikum Bergmannstrost Halle E-Mail: markus.heinecke@bergmannstrost.de

\section{Bibliografie}

DOI https://doi.org/10.1055/a-0647-2670 OP-JOURNAL 2018; 34: 186-187 @ Georg Thieme Verlag KG Stuttgart · New York ISSN 0178-1715 